\title{
Modification of wetting properties of laser-textured surfaces by depositing triboelectrically charged Teflon particles
}

\author{
Ilker S. Bayer • Fernando Brandi • Roberto Cingolani • \\ Athanassia Athanassiou
}

Received: 23 July 2012 / Accepted: 29 July 2012 /Published online: 18 August 2012

(C) Springer-Verlag 2012

\begin{abstract}
Hydrophilic laser-textured silicon wafers with natural oxide surfaces were rendered hydrophobic by depositing electrostatically charged submicrometer Teflon particles, a process termed as triboelectric Teflon adhesion. Silicon surfaces were micro-textured $(\sim 5 \mu \mathrm{m})$ by laser ablation using a nanosecond pulsed UV laser. By varying laser fluence, micro-texture morphology of the wafers could be reproduced and well controlled. Wetting properties of the triboelectrically charged Teflon-deposited surfaces were studied by measuring apparent static water contact angles and water contact angle hysteresis as a function of substrate roughness and the amount of Teflon deposited. A similar study was also performed on various micro-textured silicon carbide surfaces (sandpapers). If the average substrate roughness is between 15 and $60 \mu \mathrm{m}$, superhydrophobic surfaces can be easily formed by Teflon deposition with water contact angle hysteresis less than $8^{\circ}$. This environmentally benign solvent-free process is a highly efficient, rapid, and inexpensive way to render contactcharged rough surfaces hydrophobic or superhydrophobic.
\end{abstract}

This article is part of the Topical Collection on Contact Angle Hysteresis

I. S. Bayer $(\bowtie) \cdot$ A. Athanassiou $(\bowtie)$

Center for Biomolecular Nanotechnologies@UNILE,

Istituto Italiano di Tecnologia (IIT),

Via Barsanti 1,

73010 Arnesano Lecce, Italy

e-mail: ilker.bayer@iit.it

e-mail: athanassia.athanassiou@iit.it

\section{S. Bayer}

Department of Mechanical and Aerospace Engineering,

University of Virginia,

122 Engineer's Way,

Charlottesville, VA 22904, USA

F. Brandi $\cdot$ R. Cingolani $\cdot$ A. Athanassiou

Italian Institute of Technology (IIT),

via Morego 30,

16152, Genoa, Italy
Keywords Laser ablation · Triboelectric polymer · Teflon · Textured surfaces $\cdot$ Superhydrophobicity

\section{Introduction}

Micro- and nanostructuring of surfaces has been extensively used recently in order to control the wetting properties of surfaces with particular emphasis on making them either superhydrophilic or superhydrophobic $[1,2]$. Such increased research interest is due to the wide range of applications in which these surfaces can be used, such as biological scaffolds, self-cleaning surfaces, microfluidics, lab-on-chip devices, coatings for automotive and aerospace vehicles, and textiles [3-5]. To generate micro- and/or nanostructures, and thus tailor the wettability of diverse surfaces, several physical and chemical patterning approaches have been employed, including photolithography, electron-beam etching, templated electrochemical deposition, plasma treatment, and selective growth of nanotubes, atomic force microscopy, soft lithography, laser micromachining, and microsphere nano-patterning [6-9]. Electron-beam etching induces high amounts of heat and potential damage to the surface, while photolithography involves multiple steps and requires the use of chemicals. Atomic force microscopy can be used to mechanically etch the surface, but the procedure is slow and is not suitable for large areas. Soft lithography techniques such as micro-contact printing, micro-molding, micro-transfer molding and replica molding, imprinting and injection molding have been extensively used due to their low thermal effect, low cost, and large area applicability [10]. However, these techniques involve multiple steps and require specialized masters. Microsphere nano-patterning, in which a laser light is focused through a layer of selfassembled microparticles, is able to form submicrometer periodic patterns but with a number of disadvantages 
including control of particle assembly and additional patterning over the processed particles.

Laser micromachining, on the other hand, has a number of advantages such as fast material processing speed, large scan area, and single-step capability [11]. Surface patterning using, for example, nano- and femtosecond lasers has many applications such as fabrication of MEMS/NEMS, CMOS, 3D-microstructures, micro-trenches, micro-channels, microholes, structured or aligned submicrometer gratings and nanophotonics as well as specialized surfaces for bacterial activity $[12,13]$. It can also be used for patterning of magnetic and transparent thin films which can be used for microelectronics applications such as fabrication of flat panel displays and hard disk drives [14]. In this work, by using the concept of triboelectric charging of polymer particles [15-17] confined between two dielectric surfaces we demonstrate a solventless, rapid and simple technique to alter the wetting properties of UV nanosecond laser-ablated silicon wafer surfaces having natural oxide layers. Robust and hydrophobic thin Teflon layers can be formed on these rough surfaces when these submicrometer particles are electrically charged using commercial cellulosic foam. Our aim was to fabricate ceramic surfaces that mimic the surface architecture of self-cleaning lotus leaf surfaces. By varying the laser fluence, it is possible to control the surface roughness of the wafers in a systematic and reproducible way and thus the final wetting properties. The laser ablation technique allowed generation of average surface roughness of the order of $5 \mu \mathrm{m}$. In order to investigate the effect of a wider surface roughness range, we have used silicon carbide sandpapers of various grit sizes as model substrates as well. We found that when average surface roughness (in the form of protruding pyramid-like features) of the substrates is between 15 and $60 \mu \mathrm{m}$, superhydrophobic surfaces can easily be made with the present technique.

\section{Experimental}

Fabrication of micro-textured silicon wafers

Commercial standard silicon wafers (one side polished) having natural oxide surface layers were used in this study. No chemical treatment or mechanical polishing was used to remove the natural oxide layers from the silicon wafers. Both polished and unpolished sides of the wafers were used to create micro-texture using laser ablation for Teflon deposition. A UV laser beam was focused using a cylindrical lens with a focal length of $75 \mathrm{~mm}$. Different laser fluences were used ranging from 0.5 and $2.0 \mathrm{~J} / \mathrm{cm}^{2}$ all under a nanosecond pulse rate. The silicon wafers were kept in liquid baths such as methanol or distilled water, with $5 \mathrm{~mm}$ of liquid layer covering them during laser ablation process. The wafers were moved in precise small steps using an $x-y$ translation stage until a textured surface region of approximately $1 \mathrm{~cm}^{2}$ area was obtained. Many such rough surfaces with approximately $1 \mathrm{~cm}^{2}$ area can be fabricated on a single silicon wafer. After ablation, the wafers were removed from the liquid baths and left to dry under ambient conditions. More details about the present laser texturing technique and various other parameters effecting silicon wafer surface structuring can be found in [18].

\section{Triboelectric deposition of Teflon on textured surfaces}

Teflon powder was purchased from Sigma-Aldrich. The powder is composed of highly agglomerated submicrometer particles with an average particle size of $\sim 150 \mathrm{~nm}$. Certain amount of Teflon powder was spread over a clean cellulosebased foam surface. A commercial Scotch Brite (3M) medium duty cellulosic foam with dimensions $15 \mathrm{~cm} \times 9 \mathrm{~cm} \times$ $2 \mathrm{~cm}$ was used. A clean metal rod was rubbed over the Teflon-deposited foam surface continuously for a few minutes to spread Teflon evenly over the foam surface. Following this, the foam was continuously rubbed against the textured silicon surfaces in circular motion for a few minutes. At the end of this process, almost all of the Teflon particles spread over the foam surface were transferred to the textured silicon surface forming a thin Teflon layer. It was found that Teflon particles were strongly attached onto the laser-formed structures, since post treatment by immersing in different solvents such as acetone, toluene, methanol, or chloroform did not cause particle removal from the surfaces. A slightly different approach was followed to generate hydrophobic/superhydrophobic surfaces on silicon carbide sandpapers. Submicrometer Teflon powder was directly deposited on the sandpapers and a clean cellulosic foam was rubbed over the surfaces directly for a few minutes to spread the Teflon particles over the rough surface. Typically, sand papers of 600,800 , and 1200 grit sizes were used as substrates. The weight of the samples was recorded before and after the Teflon deposition in order to find a possible correlation between the amounts of Teflon adhered to the surfaces and the resultant wetting characteristics.

\section{Contact angle measurements}

Wettability tests (sessile drop method) were performed using a video-based optical contact angle-measuring system (Kruss GmbH, Germany). A tilting plate method was used to measure the advancing $\left(\theta_{\mathrm{A}}\right)$ and receding $\left(\theta_{\mathrm{R}}\right)$ water contact angles on textured surfaces as well on Teflondeposited surfaces. A water droplet was placed on the target surfaces, and then the sample was slowly tilted while a CCD camera recorded changes in the droplet's shape. $\theta_{\mathrm{A}}$ and $\theta_{\mathrm{R}}$ 
were measured using the last recorded image before the droplet base started to move. All contact angles were measured automatically using the software of the contact anglemeasuring system. Measurements were performed under ambient laboratory conditions using water droplets of 10 $12 \mu \mathrm{L}$ volume. All data are given as an average value from at least ten independent measurements including $\pm 3^{\circ}$ standard deviation.

\section{Characterization of the surfaces}

The morphology of the laser-textured surfaces was inspected with scanning electron microscopy (SEM) and atomic force microscopy (AFM) in non-contact mode. After Teflon deposition, surface micro- and nano-textures were mainly analyzed with AFM. In general, between 5 and $20 \mu \mathrm{m}$ scan sizes were used with simultaneous acquisition of topography, height, error and phase images. A Park Systems XE-100 AFM is used in non-contact mode with a silicon cantilever. An adaptive scan rate between $0.15 \mathrm{~Hz}$ and $0.25 \mathrm{~Hz}$ was utilized for all samples.

\section{Results and discussion}

The laser patterning technique used in this study is ideally suited for the generation of micrometer sized protruding structures on polished or unpolished silicon wafers. Figure 1 shows the SEM images of the laser-patterned surfaces obtained on the polished surface of a silicon wafer. Densely packed pyramid-like roughness features (pillars with pillar density of $0.8 \mathrm{pillar} / \mu \mathrm{m}$ ) of the order of $2 \mu \mathrm{m}$ in size were produced on the silicon wafer surface. These surfaces were hydrophilic. Finite water contact angles could not be measured on the textured surfaces and for all practical purposes an apparent water contact angle of $0^{\circ}$ was assigned to these surfaces. In Fig. 2, AFM topographical and error signal images are shown. Note that highquality AFM image acquisition of surfaces with micrometer features is only possible when the deflection (or amplitude) signals are minimized. This is because the deflection and amplitude images are the error signals in AFM. If these signals are at a minimum, the error images obtained during scanning should look identical to topographical images. Indeed, both the topographical and the error signal images in Fig. 2 match perfectly, indicating that there exist no additional phases or impurities on the laser-textured surface as a result of laser exposure. Moreover, from these images, it is seen that, practically, there exists no single and isolated pyramidal roughness feature but rather the surface topography is made up of collective pyramidal structures which probably were carved out of larger surface bumps during laser ablation.

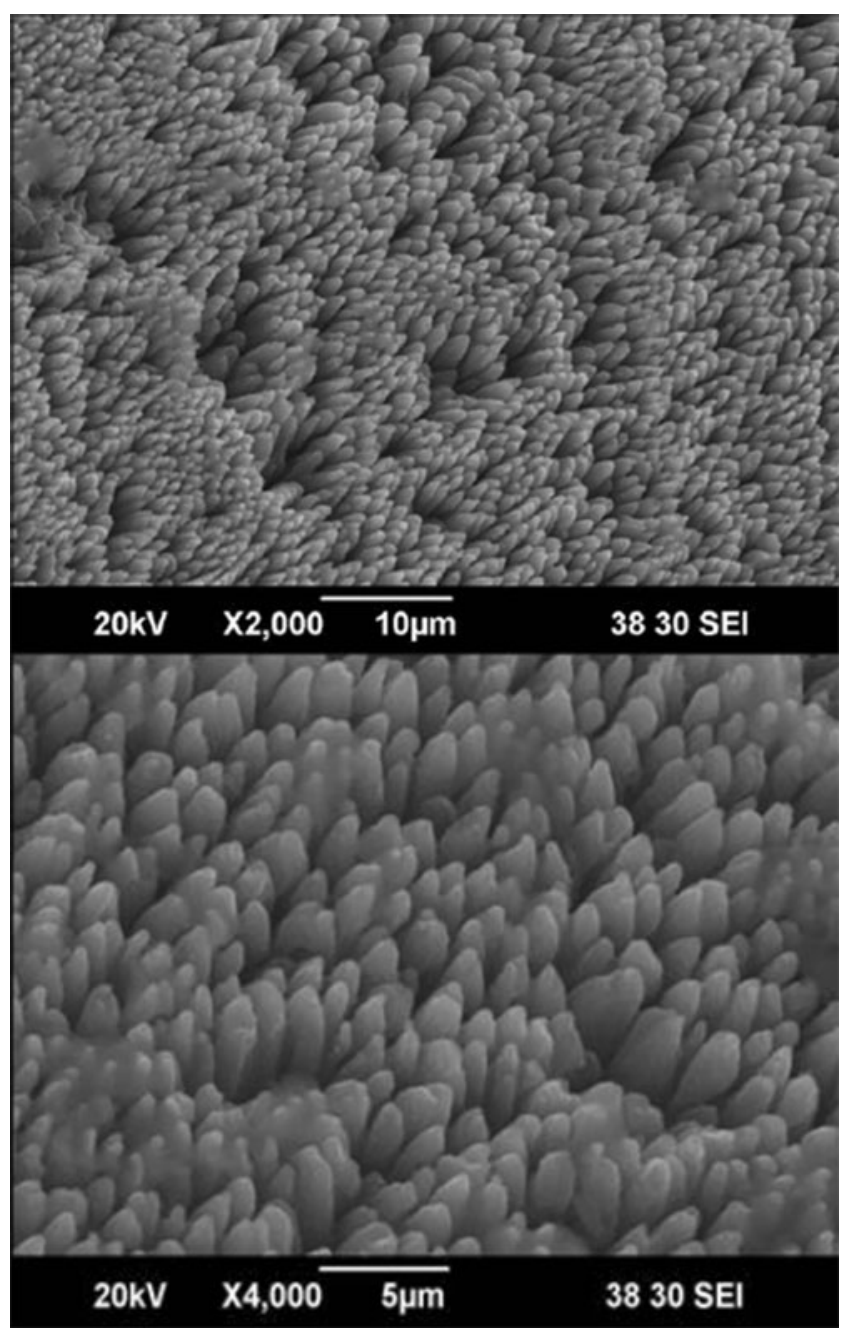

Fig. 1 Scanning electron microscope (SEM) images (top low magnification; bottom high magnification) from a laser-ablated area on a silicon wafer. Closely packed pyramid-like roughness features of average size of $2 \mu \mathrm{m}$ can be distinguished

Figure 3 displays a high-magnification AFM topography of the patterned surface corresponding to Fig. 2 using a UV nanosecond laser at a fluence of $1.0 \mathrm{~J} / \mathrm{cm}^{2}$. Detailed morphology of the pyramid-like roughness features is very clear in this AFM image.

It is seen that four pyramid-like roughness features are packed into a $25 \mu^{2}$ are on the silicon surface. Also, the base of the pyramids is made up of a layered structure most probably originating from laser induced meltingsolidification cycles [13]. Roughness analysis indicates that the base of the pyramids is about $2 \mu \mathrm{m}$ wide and the top is $0.5 \mu \mathrm{m}$ wide. This surface morphology namely, the size, depth and the frequency of distribution of the resultant protruding features, strongly depends on the laser fluence and the application technique. In the present case, the samples are prepared by irradiating the wafers with a line focus beam of about $20 \mathrm{~mm}$ long and $0.2 \mathrm{~mm}$ wide while rotating the wafer. The line focus is set radially starting slightly from 
Fig. 2 AFM topographical (a) and error signal images (b) corresponding to a lasertextured surface using the UV nanosecond laser at a fluence of $1.0 \mathrm{~J} / \mathrm{cm}^{2}$

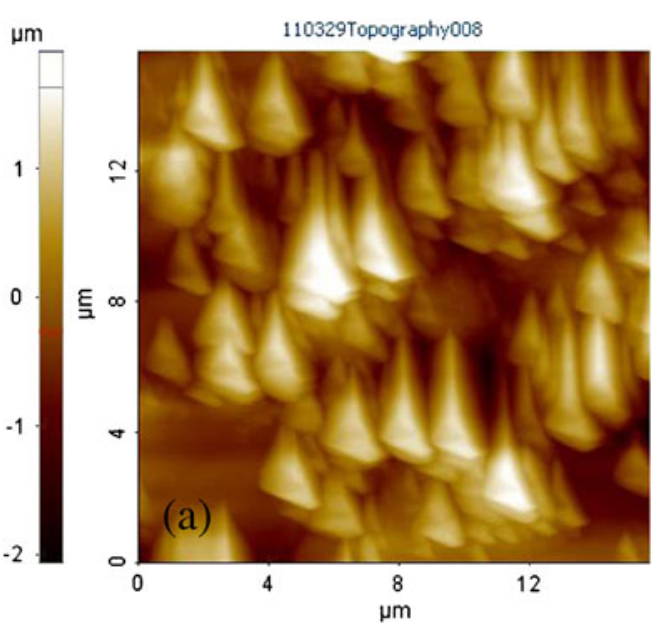

the center of the wafer, thus the actual shot dose, i.e., number of laser shots per unit surface area, is changing along the radius. The wetting of these surfaces was characterized as superhydrophilic, regardless of the applied laser fluence range. In fact, water droplets placed on these surfaces showed an apparent contact angle of $25^{\circ}$ immediately after deposition; however, after a few seconds they
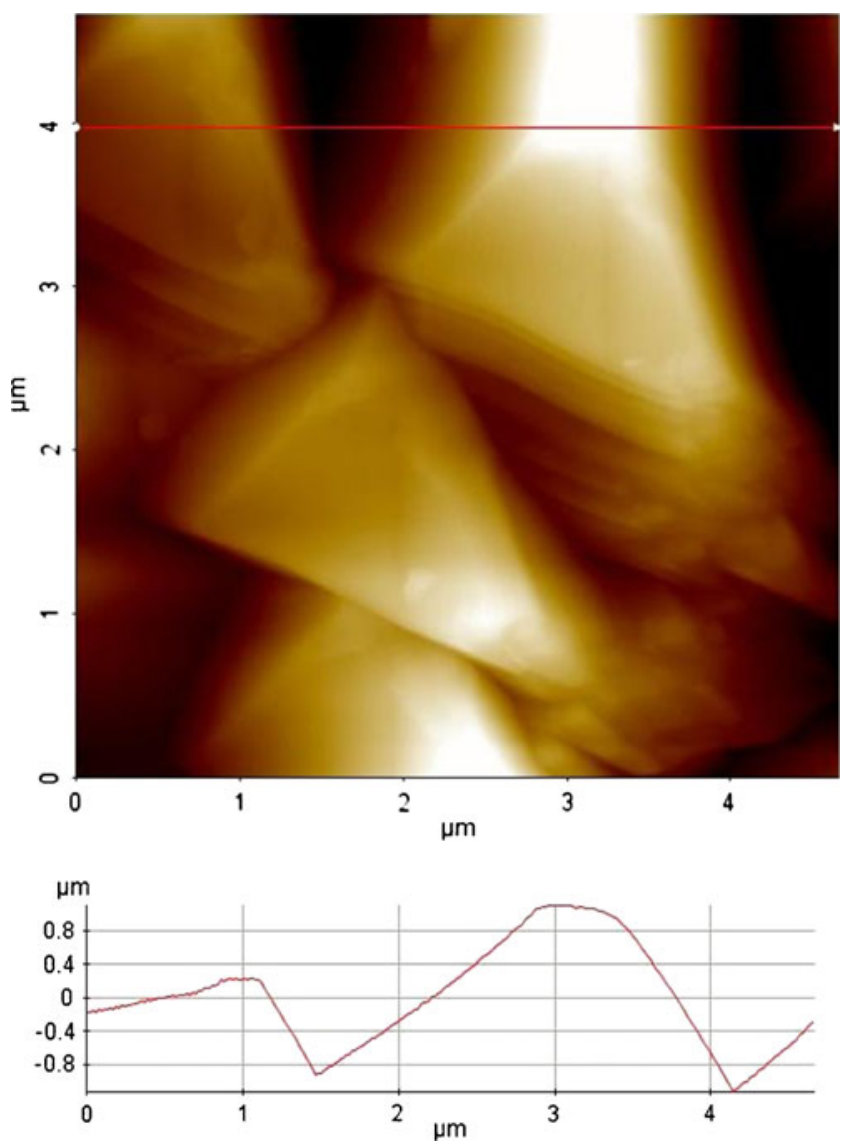

Fig. 3 Top AFM topography of a $5 \times 5 \mu \mathrm{m}$ area of the laser microtextured silicon wafer surface. Bottom Typical roughness profile of the micro-textured surface completely spread on the textured surfaces showing no finite contact angle.

When submicrometer Teflon particles are adhered to the surface by the triboelectric (contact friction) charging method described here, the surface morphology as well as the wetting of the original surface changes drastically due to the deposition of Teflon particles into the laserformed asperities. Two main factors were found to influence the final composite surface wetting characteristics, namely, the substrate micro-roughness and the amount of Teflon adhered. Submicrometer Teflon particles used in the present study were well characterized and have been recently used in the fabrication of functional superhydropohic polymer nanocomposites [19-22]. Figure 4 shows morphology of as-received Teflon powder. As-received powder is made up of agglomerated submicrometer Teflon particles as seen in Fig. 4. Figure 5 shows two 3D AFM topographic images of an untreated laser-textured surface and a triboelectrically deposited Teflon surface, respectively.

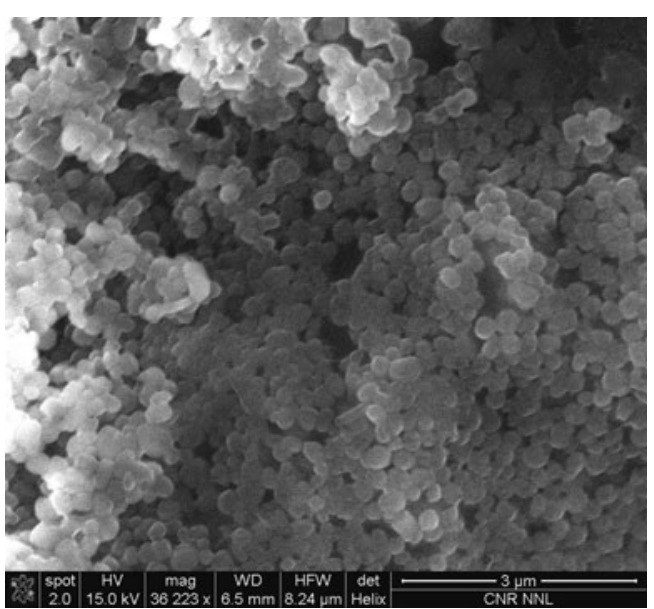

Fig. 4 Scanning electron microscope (SEM) image of the as-received Teflon powder before triboelectric charging for surface deposition 
Fig. 5 a A 3D AFM

topography (large area scan) of laser-textured silicon wafer surface before triboelectric Teflon adhesion and $\mathbf{b}$ 3D topographical detail of the resultant surface roughness after Teflon deposition. The resulting apparent water contact angle on the surface shown in (b) was measured to be $135^{\circ}$
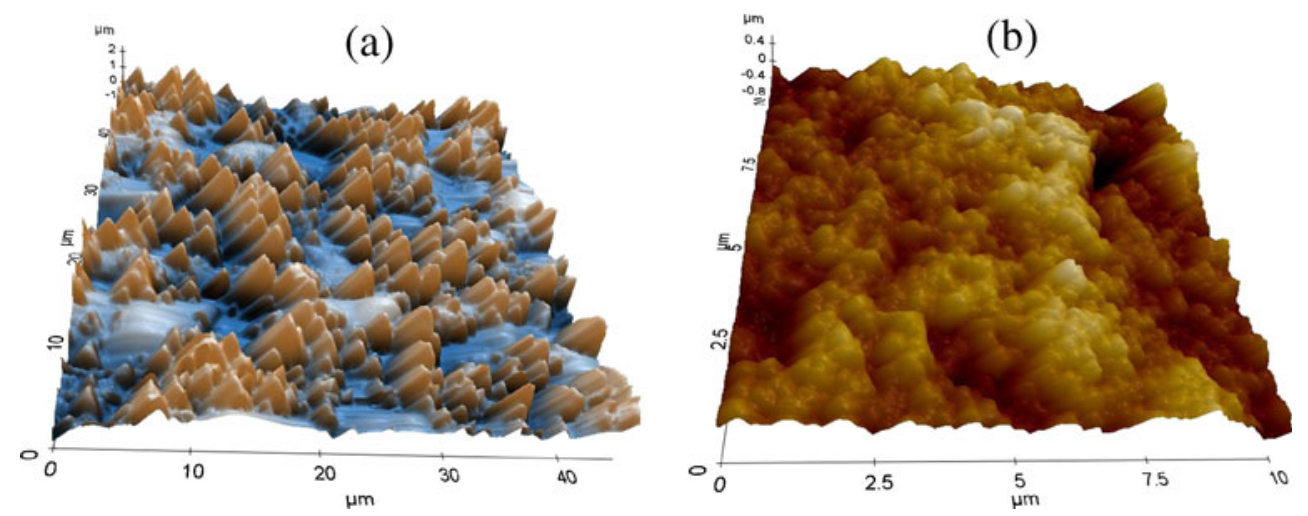

The composite hydrophobic surface shown in Fig. 5b displayed an apparent water contact angle of $135^{\circ}$ with contact angle hysteresis of $25^{\circ}$.

Since Teflon is intrinsically hydrophobic, the Tefloncoated surface also becomes hydrophobic but due to the underlying roughness the static contact angles exceed that of smooth Teflon surface which is around $110^{\circ}$. Detailed static water contact angle and contact angle hysteresis measurements showed that laser-textured silicon surfaces having an average surface roughness of $\sim 5 \mu \mathrm{m}$ do not become superhydrophobic (contact angles $>150^{\circ}$ ) when coated with triboelectrically charged Teflon particles. Composite hydrophobic surfaces obtained this way showed relatively high contact angle hysteresis compared to self-cleaning superhydrophobic surfaces $\left(<10^{\circ}\right)$ which on average was around $35^{\circ}$. In Fig. 6, detailed static water contact angle and contact angle hysteresis measurements are presented for Tefloncoated surfaces. Four different textured surfaces were used as the substrates as a result of four different laser fluences $\left(0.5,1.0,1.5\right.$ and $\left.2.0 \mathrm{~J} / \mathrm{cm}^{2}\right)$. Within the fluence range studied, no major changes in the silicon wafer surface roughness were observed although the shape of the individual pyramid-like micro-features could be modified. However, this had no direct effect on the final wetting properties of the Teflon-coated surfaces. As seen, apparent static water contact angles center around $130^{\circ}$ for all surfaces on average. Contact angle hysteresis, on the other hand, is approximately $35^{\circ}$ on average. Although, these surfaces lack the ability to self-clean, indicated by the water contact angle measurements being less than $150^{\circ}$ and contact angle hysteresis being greater than $10^{\circ}$, water droplets can still slide off the surfaces when they are adequately tilted $\sim 40^{\circ}$. This wetting property of the composite surfaces outperforms certain hydrophobic polymers such as smooth Teflon or poly(dimethyl siloxane) (PDMS). Smooth PDMS surfaces have high contact angle hysteresis close to $90^{\circ}$.

Within the laser fluence range studied, solventless triboelectric Teflon deposition technique results in surfaces with almost identical hydrophobicity. The main reason for this is attributed to the fact that the triboelectric coating creates about $1.5-\mu m$-thick Teflon films with the 150-nm Teflon powder. Therefore, a conformal coating was not possible with the resultant silicon wafer surface roughness range (2-5 $\mu \mathrm{m})$ towards forming a hierarchial surface roughness necessary for superhydrophobicity.

In order to circumvent this problem, we have used silicon carbide sandpapers of various grit sizes to analyze the effect of surface micro-texture on the degree of final hydrophobicity as
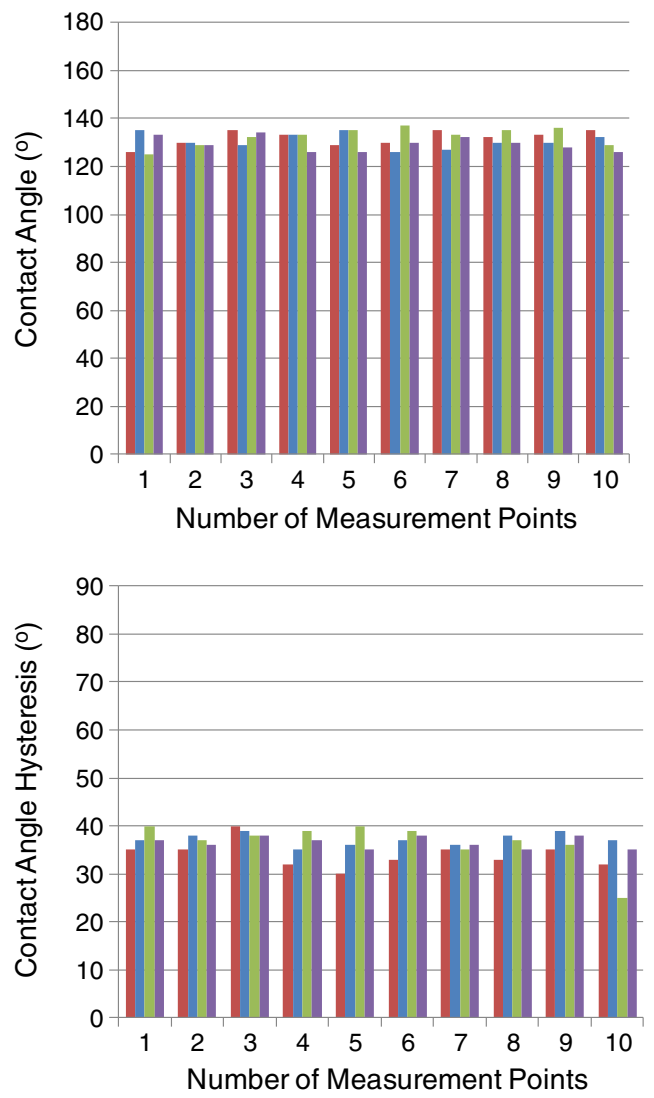

Fig. 6 Static water contact angle (top panel) and water contact angle hysteresis on Teflon-coated laser-textured silicon wafers. Different colors in the graphs indicate surfaces created using different laser fluences ranging from 0.5 to $2.0 \mathrm{~J} / \mathrm{cm}^{2}$. Ten random measurements were taken on each surface to investigate the wetting homogeneity on the surfaces 


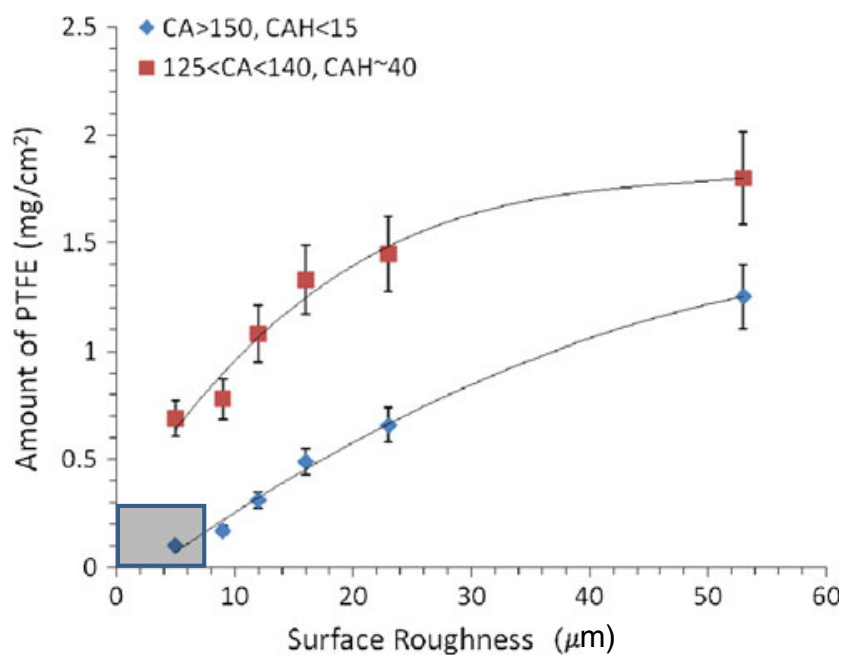

Fig. 7 Effect of amount of Teflon (PTFE) deposition on the degree of hydrophobicity as a function of surface roughness. The gray box indicates the roughness scales which could be fabricated on smooth silicon wafer surfaces with the present UV laser ablation technique. Higher surface roughness scales outside of this region are due to use of silicon carbide sandpapers of different grit sizes. The data set given $\left(\mathrm{CA}>150^{\circ}, \mathrm{CAH}<15^{\circ}\right)$ indicates self-cleaning superhydrophobicity

a result of triboelectric Teflon particle deposition. Microtextured $\mathrm{SiC}$ surfaces such as sandpapers have been recently used to fabricate highly conductive polymeric surfaces as well as oil repellent nanostructured polymer composites $[15,23]$. Figure 7 shows that depending on the amount of Teflon adhered per unit area, surface hydrophobicity can be tuned from hydrophobic to self-cleaning superhydrophobic as a function of surface micro-roughness. In general, for microrough surfaces up to $60 \mu \mathrm{m}$, surface roughness less than $1.5 \mathrm{mg} / \mathrm{cm}^{2}$ Teflon attachment is enough to render them superhydrophobic. The rectangular region in Fig. 7 indicates the roughness range which could be created by our UV laser ablation technique on silicon wafer surfaces compared to

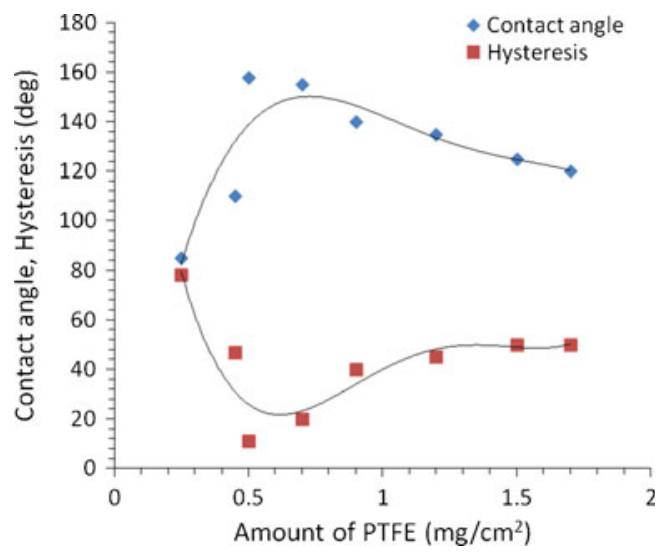

Fig. 8 Effect of amount of submicrometer Teflon deposited on a sandpaper with an average roughness of $16 \mu \mathrm{m}$ on the degree of final hydrophobicity expressed in terms of apparent water static contact angles and contact angle hysteresis. PTFE measurements were accurate to $0.1 \mathrm{mg} / \mathrm{cm}^{2}$

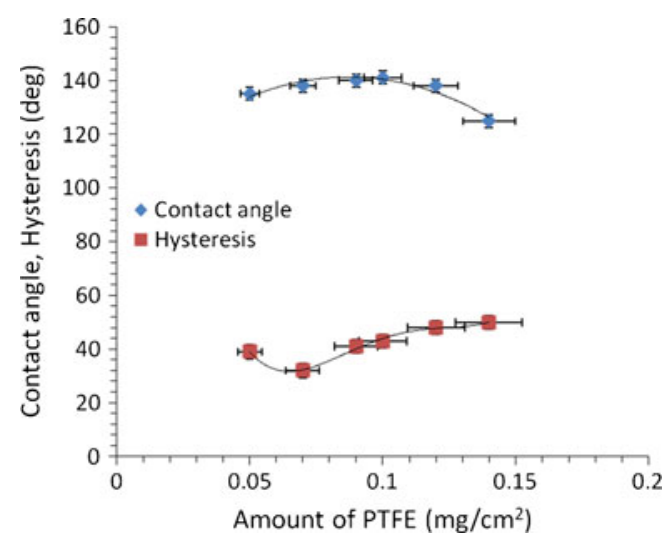

Fig. 9 Effect of amount of submicrometer Teflon deposited on a lasertextured surfaces with an average roughness of $2 \mu \mathrm{m}$ on the degree of final hydrophobicity expressed in terms of water static contact angles and contact angle hysteresis

typical commercial sand paper roughness grits. In Fig. 8, we show the changes in water contact angle and contact angle hysteresis as function of submicrometer Teflon deposited on a sandpaper having an average roughness of $16 \mu \mathrm{m}$. Best results (water contact angles exceeding $155^{\circ}$ and contact angle hysteresis less than $20^{\circ}$ ) are obtained when approximately 0.5 $0.7 \mathrm{mg}$ Teflon per $\mathrm{cm}^{2}$ is deposited on the surface.

A similar analysis conducted on laser micro-textured silicon wafer surface shows that much less amount of Teflon per unit area $\left(0.05-0.15 \mathrm{mg} / \mathrm{cm}^{2}\right)$ is necessary to render the surface hydrophobic as shown in Fig. 9. As seen, the overall changes in both the apparent water contact angles and contact angle hysteresis due to the amount of Teflon deposition is not as significant as the silicon carbide sandpaper surfaces with higher roughness scales as shown in Fig. 8.

Finally, in Fig. 10, a photograph of a sandpaper (600 grit; average roughness, $26 \mu \mathrm{m}$ ) part of which was coated with the triboelectric Teflon deposition method is shown. In the photograph, the red line shows the border between

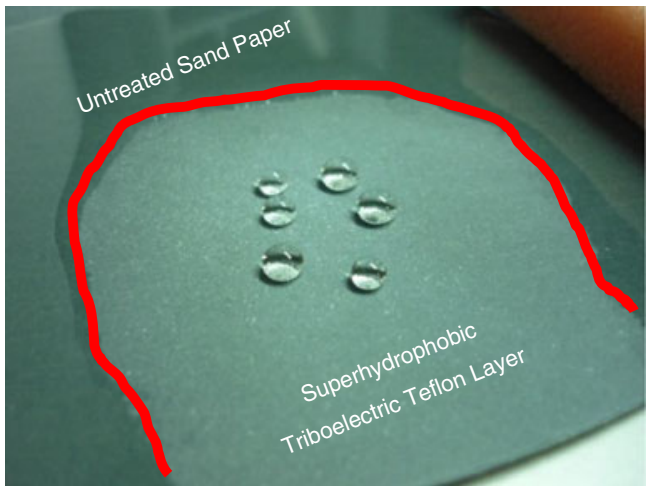

Fig. 10 Photograph of 600-grit silicon carbide sand paper part of which was rendered superhydrophobic by the triboelectric Teflon deposition method (inner region encircled by the red line). The untreated region is completely wetted by water whereas water droplets on the treated region is in a superhydrophobic state 
the treated and the untreated regions. The untreated (hydrophilic) region was completely wetted by water whereas the treated region is completely superhydrophobic. The surface roughness $(\sim 26 \mu \mathrm{m})$ corresponding to this 600 grit silicon carbide sandpaper is within the roughness range 15$65 \mu \mathrm{m}$ which is considered ideal for forming superhydrophobic surfaces with the present solventless triboelectric Teflon deposition method.

\section{Conclusions}

Using the principle of triboelectric charging of insulating polymers, we have demonstrated a solvent- and chemical treatmentfree technique to render rough surfaces hydrophobic and superhydrophobic with low contact angle hysteresis. Two types of rough surfaces were employed: (a) micro-machined $(\sim 5 \mu \mathrm{m})$ silicon wafers using a nanosecond UV laser and (b) coarse $(>16 \mu \mathrm{m})$ silicon carbide sandpapers of various grit sizes. These surfaces were superhydrophilic before the treatment. Submicrometer Teflon particles were initially spread on these rough surfaces in a coarse manner and then a clean cellulosic foam surface was rubbed against the Teflon-deposited surfaces to smooth and spread out the particles, and at the same time electrostatically charging them. These particles adhered strongly to the textured surfaces due to electronic charging during rubbing. Depending on the underlying surface roughness, the surfaces could also be rendered superhydrophobic. Lasertextured surfaces did not achieve superhydrophobicity due to their low surface roughness $(\sim 5 \mu \mathrm{m})$. Contact angles as high as $130^{\circ}$ were measured on these surfaces with contact angle hysteresis around $35^{\circ}$. On the commercial sandpaper surfaces with higher surface roughness, superhydrophobicity can be achieved only if the surface micro-roughness is within a certain range which was found to be 15-60 $\mu \mathrm{m}$.

\section{References}

1. Zorba V, Persano L, Pisignano D, Athanassiou A, Stratakis E, Cingolani R, Tzanetakis P, Fotakis C (2006) Making silicon hydrophobic: wettability control by two-lengthscale simultaneous patterning with femtosecond laser irradiation. Nanotechnology 17:3234-3238

2. Steele A, Bayer I, Moran S, Cannon A, King WP, Loth E (2010) Conformal $\mathrm{ZnO}$ nanocomposite coatings on micro-patterned surfaces for superhydrophobicity. Thin Solid Films 518:5426-5431

3. Nakajima A, Fujishima A, Hashimoto K, Watanabe T (1999) Preparation of transparent superhydrophobic boehmite and silica films by sublimation of aluminum acetylacetonate. Adv Mater 11:1365-1368

4. Yoshimitsu Z, Nanajima A, Watanabe T, Hashimoto K (2002) Effects of surface structure on the hydrophobicity and sliding behavior of water droplets. Langmuir 18:5818-5822

5. Patankar NA (2003) On the modeling of hydrophobic contact angles on rough surfaces. Langmuir 19:1249-1253
6. Shirtcliffe NJ, Aqil S, Evans C, McHale G, Newton MI, Perry CC, Roach P (2004) The use of high aspect ratio photoresist (SU-8) for super-hydrophobic pattern prototyping. J Micromech Microeng 14:1384-1389

7. Huang L, Lau SP, Yang HY, Leong ESP, Yu SF (2005) Stable superhydrophobic surface via carbon nanotubes coated with a $\mathrm{ZnO}$ thin film. J Phys Chem B 109:7746-7748

8. Rizzello L, Shankar SS, Fragouli D, Athanassiou A, Cingolani R, Pompa PP (2009) Microscale patterning of hydrophobic/hydrophilic surfaces by spatially controlled galvanic displacement reactions. Langmuir 25:6019-6023

9. Bartlett PN, Baumberg JJ, Birkin PR, Ghanem MA, Netti MC (2002) Highly ordered macroporous gold and platinum films formed by electrochemical deposition through templates assembled from submicron diameter monodisperse polystyrene spheres. Chem Mater 14:2199-2208

10. Biswas A, Bayer IS, Biris AS, Wang T, Dervishi E, Faupel F (2012) Advances in top-down and bottom-up surface nanofabrication: techniques, applications \& future prospects. Adv Colloid Interf Sci 170:2-27

11. Etzion I (2005) State of the art in laser surface texturing. J Tribol 127:248-254

12. Fadeeva E, Truong VK, Stiesch M, Chichkov BN, Crawford RJ, Wang J, Ivanova EP (2011) Bacterial retention on superhydrophobic titanium surfaces fabricated by femtosecond laser ablation. Langmuir 27:3012-3019

13. Leitz KH, Redlingshöfer B, Reg Y, Otto A, Schmidt M (2011) Metal ablation with short and ultrashort laser pulses. Phys Procedia 12:230-238

14. Kumar GR, Carvajal JJ, Pujol MC, Mateos X, Grau J, Massons J, Vázquez de Aldana JR, Méndez C, Moreno P, Roso L, Ferré-Borrull J, Pallarès J, Marsal LF, Aguiló M, Díaz F (2011) Surface ablation of $\mathrm{RbTiOPO}_{4}$ by femtosecond laser. Opt Mater 34:207-214

15. Bayer IS, Caramia V, Biswas A, Cingolani R, Athanassiou A (2012) Metal-like conductivity exhibited by triboelectrically deposited polyaniline (emeraldine base) particles on microtextured SiC surfaces. Appl Phys Lett 100:201604-201609

16. Calcagnile P, Fragouli D, Bayer IS, Anyfantis GC, Martiradonna L, Cozzoli PD, Cingolani R, Athanassiou A (2012) Magnetically driven floating foams for the removal of oil contaminants from water. ACS Nano 6:5413-5419

17. Liu C, Bard AJ (2008) Electrostatic electrochemistry at insulators. Nat Mater 7:505-509

18. Brandi F, Burdet N, Carzino R, Diaspro A (2010) Very large spot size effect in nanosecond laser drilling efficiency of silicon. Opt Express 18:23488-23494

19. Bayer IS, Fragouli D, Martorana PJ, Martiradonna L, Cingolani R, Athanassiou A (2011) Solvent resistant superhydrophobic films from self-emulsifying carnauba wax-alcohol emulsions. Soft Matter 7:7939-7943

20. Tiwari MK, Bayer IS, Jursich GM, Schutzius TM, Megaridis CM (2010) Highly liquid-repellent, large-area, nanostructured poly(vinylidene fluoride)/poly(ethyl 2-cyanoacrylate) composite coatings: particle filler effects. ACS Appl Mater Interfaces 2:1114-1119

21. Bayer IS, Fragouli D, Attanasio A, Sorce B, Bertoni G, Brescia R, Di Corato R, Pellegrino T, Kalyva M, Sabella S, Pompa PP, Cingolani R, Athanassiou A (2010) Water-repellent cellulose fiber networks with multifunctional properties. ACS Appl Mater Interfaces 3:4024-4031

22. Bayer IS, Caramia V, Fragouli D, Spano F, Cingolani R, Athanassiou A (2012) Electrically conductive and high temperature resistant superhydrophobic composite films from colloidal graphite. J Mater Chem 22:2057-2062

23. Das A, Schutzius TM, Bayer IS, Megaridis CM (2012) Superoleophobic and conductive carbon nanofiber/fluoropolymer composite films. Carbon 50:1346-1354 